\title{
Application of topology optimization to the tibial osteotomy fixation plates
}

\author{
M. Akif Kutuk* and Ibrahim Gov \\ Mechanical Engineering Department, Engineering Faculty, University of Gaziantep, Gaziantep, Turkiye
}

\begin{abstract}
Application of topology optimization to fixation plates is the main consideration of this paper. The interbody fusion plates are required to give mechanical support to tibia with minimally invasive surgical procedure. Topology optimization is used to obtain fixation plates with possible minimum material usage. Topology optimization is applied to three types of plates which are used in upper tibial osteotomy. Initial design of the plates are first numerically modelled and then investigated for stresses under possible highest load values. The results of the analysis indicated that the plates are very stiff even under high loads. Application of topology optimization to plates yielded minimized weight and material usage while keeping the plates still adequate for possible high load values. It was also revealed that up to $50 \%$ of mass could be saved by an optimal implant design.
\end{abstract}

Keywords: Topology optimization, finite element method, plate design, tibial osteotomy

\section{Introduction}

Topology optimization is sometimes referred to as layout optimization in the related literature and is a special form of shape optimization. The goal in the process is to find the best use of material for a body where an objective criterion (i.e., global stiffness) takes out a maximum or minimum value subject to given constraints (i.e., volume reduction). Topology optimization is trying to find preliminary, sometimes completely innovative, structural configurations that meet specific conditions [25]. Optimization of the geometry and topology of structural lay-out has great impact on the performance of structures, and the last decade has seen great amount of work in this important area of structural optimization [4].

Application of topology optimization can be observed in various application areas such as solid

\footnotetext{
*Corresponding author: Dr. M. Akif Kutuk, Mechanical Engineering Department, Engineering Faculty, University of Gaziantep, 27310 Gaziantep, Turkiye. Tel.: +90 342317 2571; Fax: +90 342 360 1104; E-mail: kutuk@gantep.edu.tr.
}

mechanics, thermal, eigen-value, and acoustic problems. It is realized that topology optimization improves performance of a structure greatly [1]. Chiandussi et al. applied topology optimization to three dimensional automotive parts [5]. Ledermann et al. used the method in aircraft structure design [19] while Larsen et al. designed micromechanisms [18]. Seo et al. developed a method based on isogeometric analysis [23]. Juan et al. combined the element free Galerkin method with evolutionary structural optimization method [15]. Application of the method is also possible in biomechanics but very small number of studies is available in the related literature. Strange et al. improved the compressive loading properties of lumbar disks by using finite element analysis [24]. Tovar et al. used topology optimisation to design plates which are used in lumbar spine fixation [25]. Chuah et al. reduced the stress shielding effect of spinal interbody by using topology optimization [6]. António et al. presented a computational model for bone remodelling around cementless (press-fit) stems with in growth control. The model was applied to a 3D FE model of an implanted femur [2]. 
A. Grujicic and M. Laberge et al. [11] investigated efficiency of nail type and plate type implants under realistic physiological loading conditions, such as four typical everyday activities namely, walking, lunging, cycling and egress.

M. Grujicic and X. Xie et al. [13] optimized a proximal radius-fracture fixation-plate by using size and shape optimization under physiological loading conditions. In the shape optimization, only outer shape of design domain is changed.

M. Grujicic and G. Arakere [12] optimized a distal femoral-fracture fixation-plate by using size optimization under realistic physiological loading conditions associated with normal living activities. In the size optimization, design domain can be optimized only changing geometrical dimensions.

Topology optimization has been used as a structural optimization method in recent years. During this process, material distribution in the design domain is optimized for possible minimum material usage. The aim is either to maximize stiffness or eigen frequency. Initially, the design domain is comprised of a large number of finite elements. The topology optimization process selectively removes some elements from the domain. Novel optimum structures are usually obtained by using this technique [25].

Bendsøe and Kikuchi developed the homogenization method for topology optimization [3]. The homogenization method is based on the modeling of a perforated material constructed from a basic unit cell consisting at a microscopic level of material and void and on the description of the structure by using a continuously varying distribution of the material density [14]. Other two approaches are density distribution method and evolutionary structural optimization (ESO) technique. The material density of each element was selected as the design variable in the density distribution method [22, 28] while low-stressed elements are gradually removed to achieve the optimal design in ESO [27]. The methods stated above have their own advantages and each one is used by many researchers.

Main disadvantage of topology optimization is solution time. To obtain final results, element number of design domain and iteration number of the solution must be increased. Therefore the process requires very long solution time. Many methods are applied to topology optimization to decrease solution time such as parallel programming [21], genetic algorithm [26] and neural network [20]. These methods are used to obtain the best solution in the shortest time. But the designer needs mathematical software (i.e. Matlab, Mathematica) to use these methods. The mathematical software are not useful for an industrialist for solid modelling and performing Finite Element Analysis (FEA) solutions as they are not customized for these applications. The use of these programs requires very long source codes for modelling and the same is true for FEA solutions as well.

In a previous study [10], element removal method (ERM) was developed to decrease solution duration of topology optimization and in this study up to $80 \%$ time reduction was obtained. The aim of this method is the elimination of passive elements (which are found from FE analyses) in the optimization processes after every iteration loop. In the next iteration loop, unnecessary elements will not be used during solution. Hence, at each progressive solution loop the number of elements are decreased, and the decrease in the element number causes decreased solution time. Flowchart of developed ERM algorithm is presented in Fig. 1.

The main goal of the present study is to emphasize the effects of ERM on plate design. This is achieved through the application of finite element based topology optimization to fixation plates which are used in upper tibial osteotomy. The application of optimization results in fixation plates with minimum material at acceptable stress values under the effect of possible highest loading values.

\section{Materials and methods}

\subsection{Topology optimization with element removal}

In this study, ERM is used to obtain topology optimization results. ERM was developed in the previous study [10] and advantages and disadvantages were investigated in that study.

Topology optimization starts with a defined design domain $(\Omega)$. The percentage of material to be eliminated (M) in $\Omega$ and material properties (Young's Modulus and Poisson's Ratio) are also defined. The theory of topology optimization seeks to minimize objective function $(\Omega-\mathrm{M})$ or maximize $(\mathrm{M})$ subject to the constraint $\left(\sigma_{\max }\right)$ defined. The design variable is von Mises stress value of each finite element $(i)$ in the topological domain. Stated in simple mathematical terms, the optimization problem is as follows for $N$ finite elements: $\mathrm{M}=$ predefined $\max$. value 


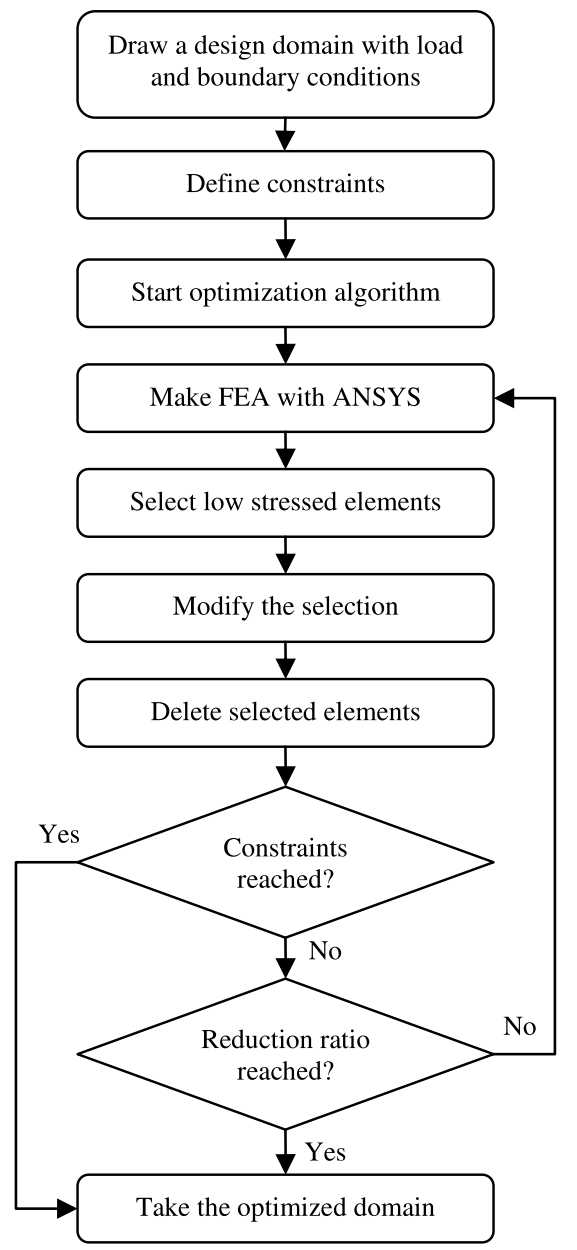

Fig. 1. Flowchart of Element Removal Method (ERM).

subject to : $\sigma_{i}<\sigma_{\max }(i=1,2,3, \ldots \ldots, N)$

In Fig. 3, a cantilever beam example is given with a single downward load $F$ at free end. Any analysis software may be used to generate the finite element (FE) model. Boundary conditions and loads are applied to the generated FE model. Strain energy of the part is

$$
U=\frac{1}{2} \int_{\text {volume }} \sigma \varepsilon d v
$$

If $N$ finite elements are used to discretize the design domain $\Omega$, then total strain energy expressed as a function of element stress, strain, elemental area $(d A)$ and element thickness $(t)$, (thickness can be considered to be constant for plane stress problem):

$$
U=\sum_{i=1}^{N} \frac{1}{2} t \int_{\text {element }} \sigma_{i} \varepsilon_{i} d A
$$

Strains may be expressed in terms of nodal displacements:

$$
\varepsilon=B q
$$

where $B$ is the strain displacement matrix, based on the element shape functions and $q$ is the nodal displacement vector. Stress, in terms of nodal displacements is expressed using material matrix $D$ :

$$
\sigma=D B q
$$

Combining equations (2), (3) and (4) the strain energy equation now becomes:

$$
\begin{aligned}
& U=\sum_{i=1}^{N} \frac{1}{2} t \int_{\text {element }} B^{T} D q^{T} B q d A \\
& U=\sum_{i=1}^{N} \frac{1}{2} q^{T} k^{e} q
\end{aligned}
$$

where $k^{e}$ is element stiffness matrix and $k^{e}=$ $t \int_{\text {element }} B^{T} D B d A$.

The FE equation may be reformulated, using the global stiffness matrix $K$, global displacement vector $Q$ and load vector $F$ in the form given below, to determine $Q$ :

$$
K Q=F
$$

Once the element displacements are calculated, strains and stresses can be determined using equations (3) and (4) respectively. The calculated stress values are used in optimization process.

In Fig. 1, a schematic view of optimization process with element removal method can be seen. With respect to this procedure firstly, design domain must be drawn with load and boundary conditions. Then, the constraints (stress, volume reduction, and etc.) are defined. After the definition of constraints the optimization process can be started. In the optimization process firstly, FEA is made and the low stressed elements are selected. Then, the modification algorithm is applied to obtain fine material distribution. After that, the selected elements are deleted and they are not used in the next iteration loops. Finally, constraints are 


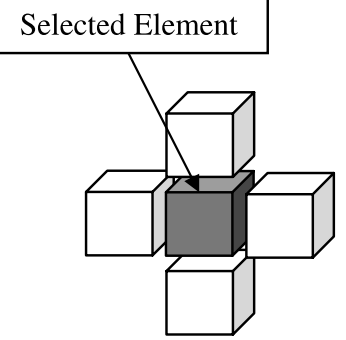

Fig. 2. Selected element and its neighbours.

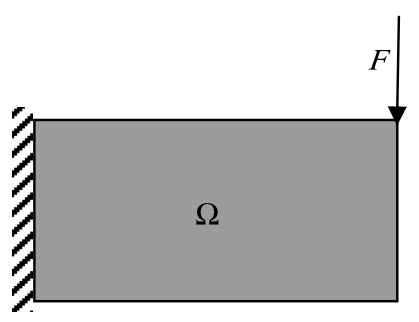

Fig. 3. Design Domain and Boundary Conditions.

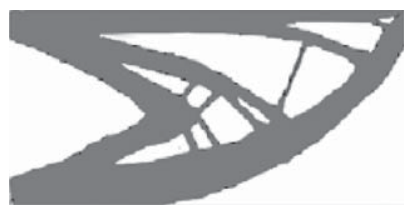

Fig. 4. Optimized Beam.

controlled, if one constraint is reached optimization procedure is finished, if not the cycles are continued.

In the modification algorithm, by looking the neighbours of the selected element (in Fig. 2), algorithm decide whether to delete or not to delete the selected element.

After applying the algorithm to the design domain in Fig. 3, material distribution given in Fig. 4 is obtained. The solution is done for $\mathrm{M}$ (mass fraction) value of $50 \%$.

Given procedure is also used to obtain optimum material distribution of fixation plates for possible minimum material usage.

\subsection{Analyses of plates}

Proximal tibia osteotomy was proven to be an effective treatment method for medial compartment osteoarthritis of the knee for young and more active elderly patients [9]. Osteotomy was fixed by three types of plates and with their four types of combination which were supported by wedges with the height of $10 \mathrm{~mm}$ (TR-2002 02021Y-Hipokrat Corp., Izmir/Turkey) and six holed anatomical "T" plates without wedges (Hipokrat Corp., Izmir/Turkey). Appearance of plates (Fig. 5a) and their mounted forms on bone (Fig. 5b) are shown in Fig. 5. The plates are modelled as shown in Fig. 6.

Axial compressive load in an adult's knee during single limb stance of fast walking was stated to be about $5600 \mathrm{~N}$ [17]. The stated value is the mean value obtained from 12 subjects ( 6 male, 6 female), ranging in weight from 40 to $90 \mathrm{~kg}$ (mean $70.8 \mathrm{~kg}$ ). In the present study, load value for design and optimization of plates is taken as $8000 \mathrm{~N}$ static loading considering heavy subjects. During stress analyses of initial forms of plates, $8000 \mathrm{~N}$ load is distributed to the holes in compressive direction, simulating the body load via screws (Fig. 7). For the anatomical T plate, load is applied from the two holes at the top and two holes at the bottom of the plate. Two mid-holes are used to fix the plate. Plates with wedges are fixed from the wedges. Materials of the plates may be steel " $316 \mathrm{~L} / 1.4441$ " and titanium "Ti-6A14V/IMI 318" [8]. Material is selected to be steel " $316 \mathrm{~L} / 1.4441$ " as yield strength value is low (about $200 \mathrm{MPa}$ ) compared to that of titanium alloy "Ti-6Al4V/IMI 318" (about $900 \mathrm{MPa}$ ). Hence, Modulus of Elasticity and Poisson's Ratio are used as $200 \mathrm{GPa}$ and 0.3 respectively. Elements used in the models are 8-noded structural and quadrilateral plane elements. ANSYS Finite Element program is used for all of the analyses.

\subsection{Optimization of plates}

The interbody fusion plates are required to give mechanical support to tibia with minimally invasive surgical procedure. During the design process of the plates, two conflicting objectives govern the design: maximize rigidity while using minimum amount of plate mass. The problem may be defined as; maximization of rigidity with minimum material usage. ERM method described above is applied to the plates, to obtain reduced volume with maximum possible stiffness. The work done is mainly static structural analysis based on FEM with linear elasticity.

The initial forms of the plates given in Fig. 6, are defined as the design domain at the beginning of the optimization process. Some design parameters; like position, number and diameters of holes, size and 
(a)
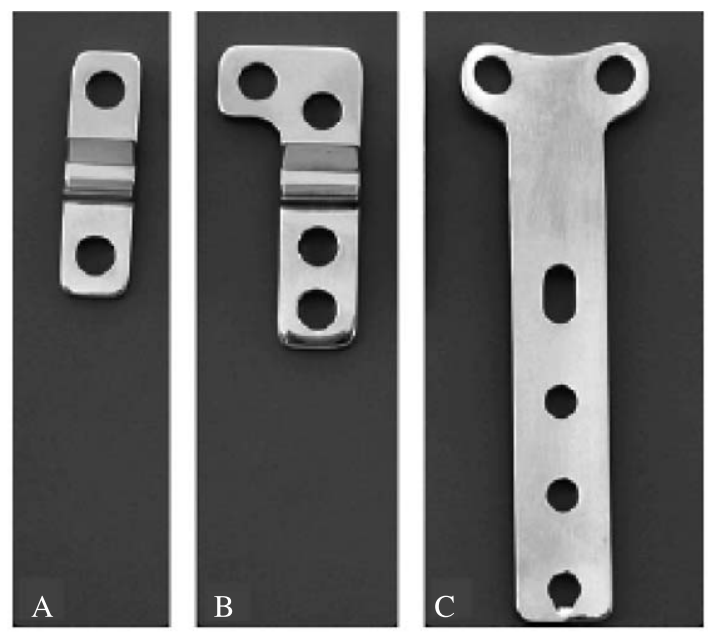

(b)
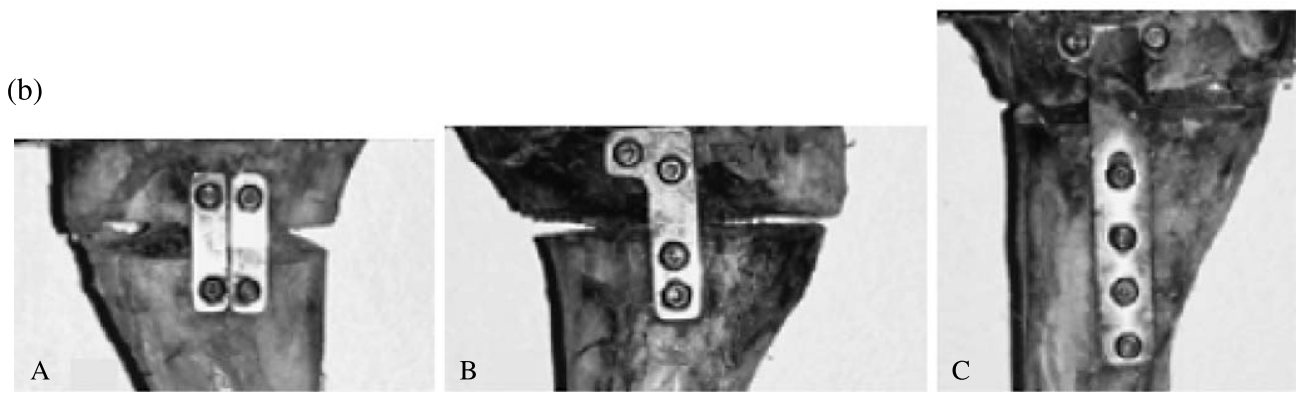

Fig. 5. Osteotomy fixation plates [9]. a. Appearance of the internal surface of plates. b. Mounted forms of plates on the bone. A: Rectangular shaped two-holed plate with wedge. B: Reversed "L" shaped four-holed plate with wedge. C: Anatomical "T" plate with 6 holes.
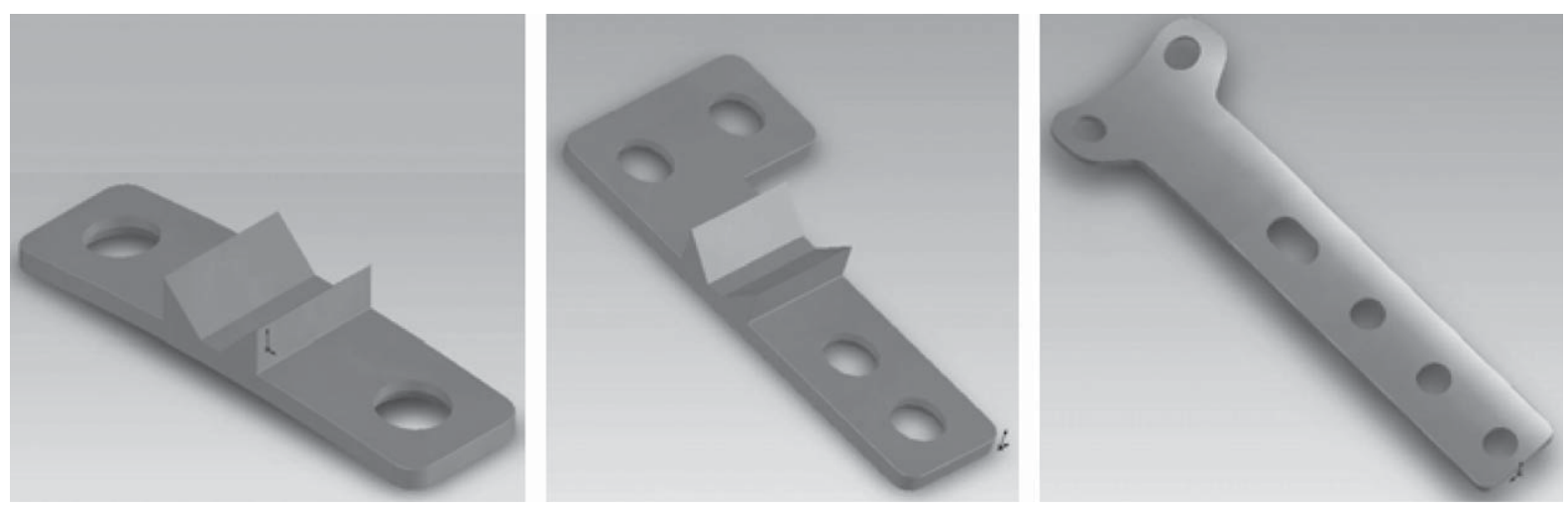

Fig. 6. Plate Models.

shapes of wedges, are considered as functional requirements of plates and no change is done on these features during the optimization loops. Plates A and B contains wedges. Considering the wedges as design requirement this region is not included in the optimization process, remaining parts of the plates can be considered 


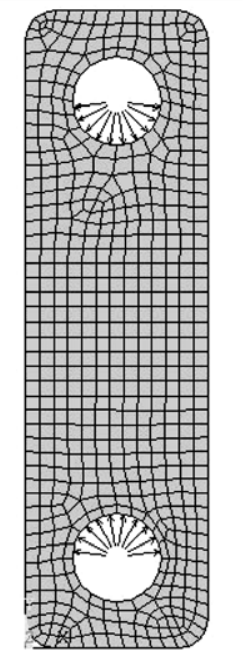

A

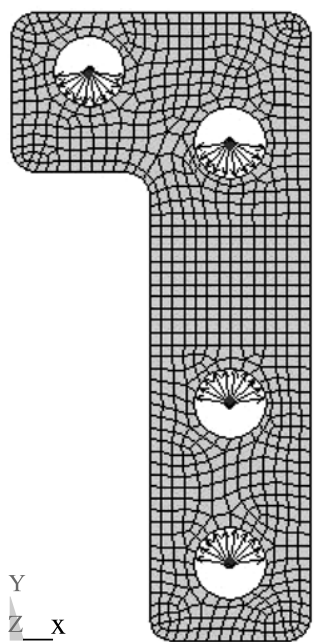

B

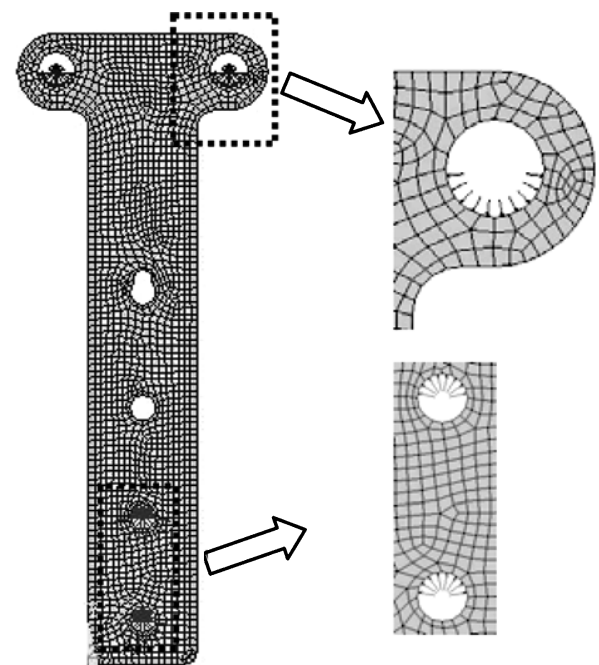

$\mathrm{C}$

Fig. 7. Meshed Models of Plates with Applied Load.

Table 1

Number of elements and nodes used in the plate design

\begin{tabular}{lccc}
\hline Plate Type: & $\mathrm{A}$ & $\mathrm{B}$ & $\mathrm{C}$ \\
\hline $\mathrm{M}(\%)$ & 42 & 36 & 50 \\
No. of Elements* & $5641 / 3227$ & $8468 / 5435$ & $18206 / 9044$ \\
$\sigma_{\max }(\mathrm{MPa})^{*}$ & $1.7 / 3.7$ & $2.7 / 3.8$ & $2.1 / 4.1$ \\
$\delta_{\max }(\mu \mathrm{m})^{*}$ & $0.05 / 0.20$ & $0.23 / 0.50$ & $0.27 / 0.70$ \\
\hline
\end{tabular}

*Values are given as (Initial Value)/(Optimized Value).

as plane stress problem with constant thickness. The design domain and FE model are given in Fig. 7 for the plates. In the figure, the plates are plotted after mesh. Number of elements for the models A, B and C are given in Table 1.

\section{Results}

\subsection{Results for analyses of the plates}

The results of analyses for von Mises stresses are given in Fig. 8 and tabulated in Table 1. In the table, maximum values of von Mises stresses $\left(\sigma_{\max }\right)$ and deformation $\left(\delta_{\max }\right)$ are given for each plate for the given load value.

Analyses indicate that all the plates are very stiff as amount of deformation under even a high load value is very small. Also the von Mises stress values are very low compared to $200 \mathrm{MPa}$ yield value.

\subsection{Results for optimization of plates}

Topology Optimization is applied to plates with the criteria of $50 \% \mathrm{M}$. Each plate is limited to allowable stress value of $5 \mathrm{MPa}$. Optimization algorithm with ERM method is used in ANSYS program for topology optimization and the flowchart is given in Fig. 1. After optimization, the plates are smoothed as shown in Fig. 9. Optimization is performed on an i5-3470 type computer, $3.2 \mathrm{GHz}$ processor with $6 \mathrm{~GB}$ RAM. Time consumed for optimizations are 57,87 and 165 seconds for plates $\mathrm{A}, \mathrm{B}$ and $\mathrm{C}$ respectively.

The effect of topology optimization is observed as reduced mass. Number of elements used for meshing of models after optimization and element number reduction ratio are given in Table 1. Increased deformation and stress values are other effects to be observed compared to those of initial shape. New values for stress and deformation are given in Table 1 for all plates. The highest mass reduction ratio is observed at plate C, T-plate, with a value of $50 \%$. Even using half of the material of initial shape, still stress and deformation values are low as seen in Table 1.

Variation of maximum von Mises stress with applied load after topology optimization is plotted in Fig. 10. In the figure, original values are also plotted for comparison. Stress values are increased on the plates after topology optimization as expected. But the new stress values are still low and hence plates are 


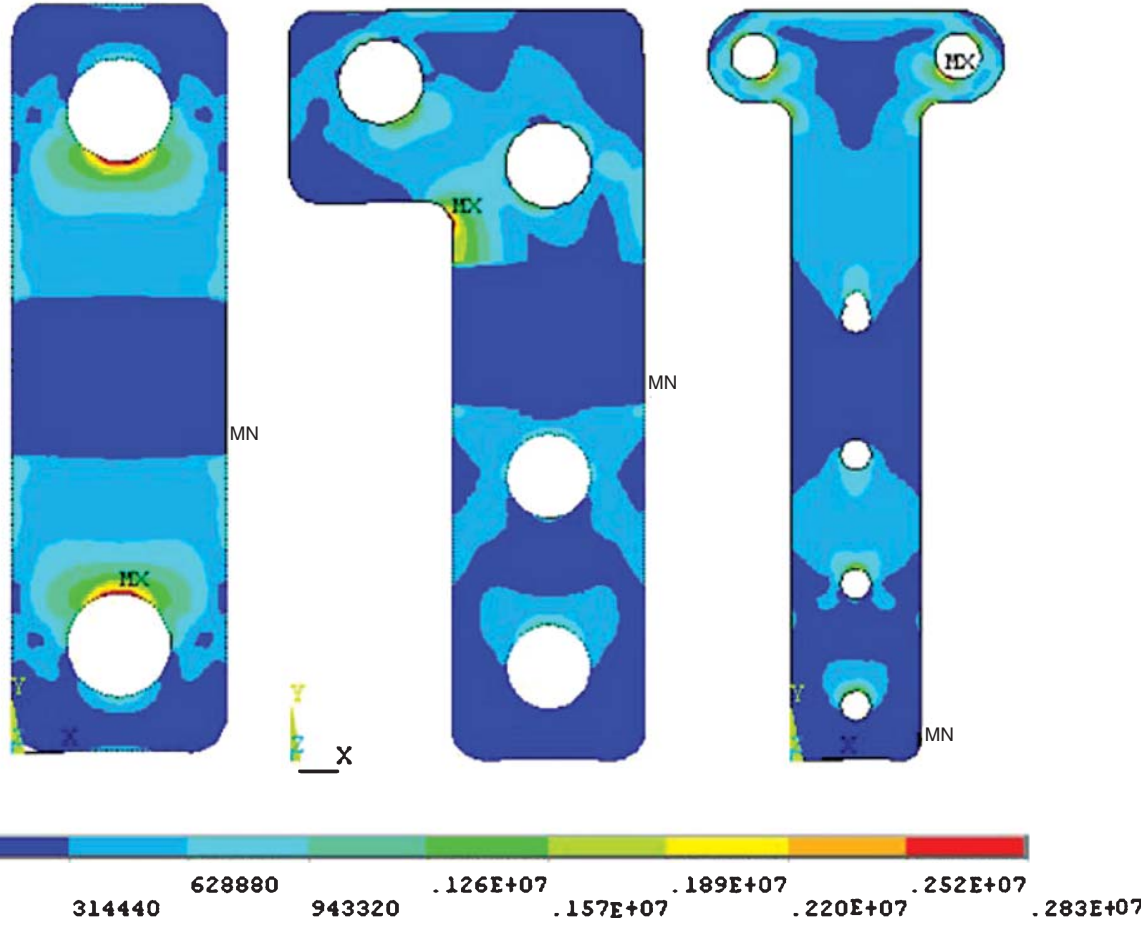

Fig. 8. Von Mises Stress Distribution on initial forms of the Plates (Deformation Scale $=1^{*} 1000$ ).

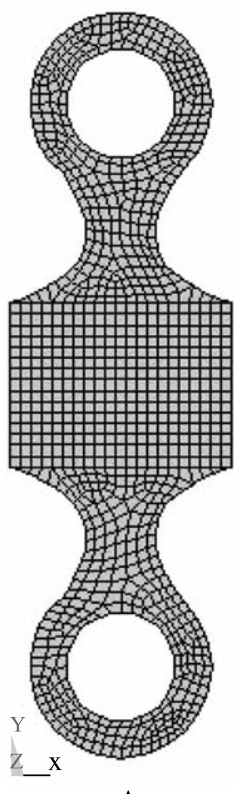

A
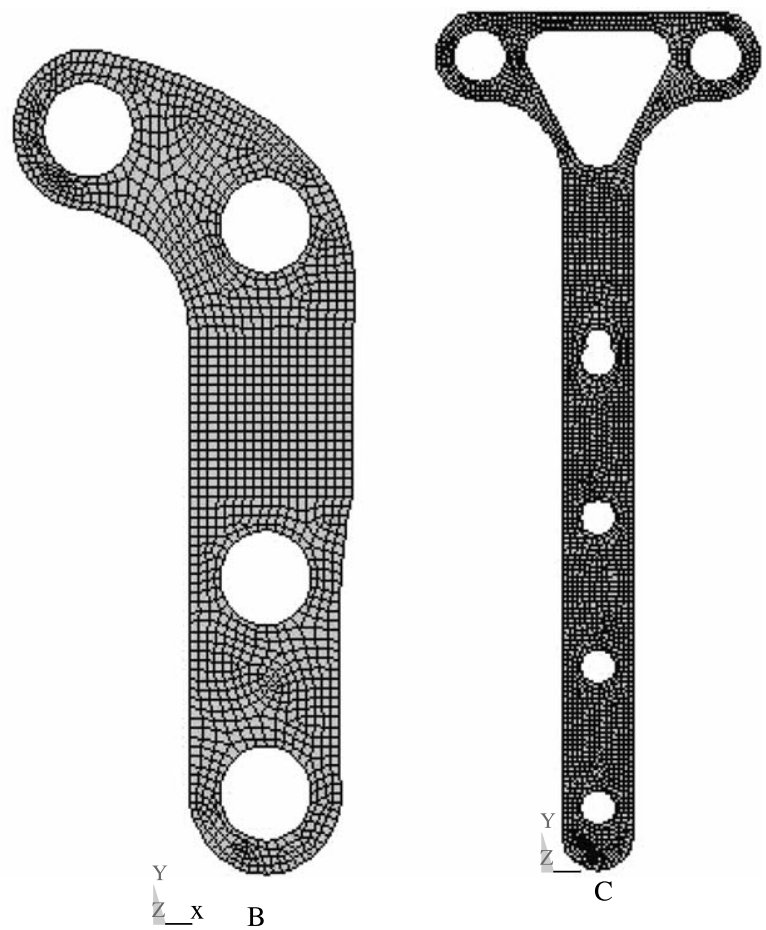

Fig. 9. Plates after Topology Optimization. 


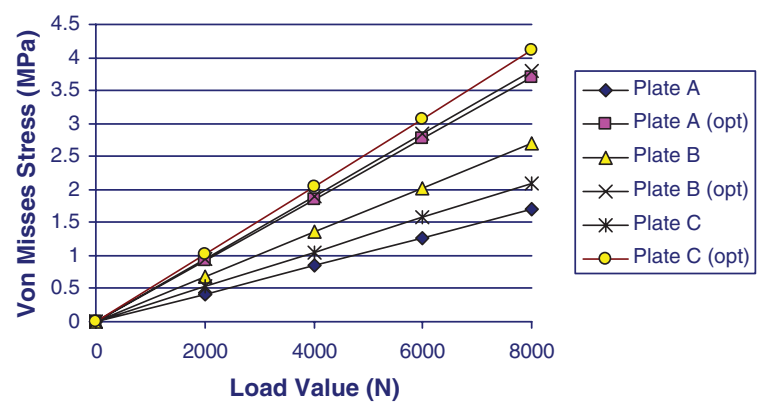

Fig. 10. Variation of Stress with Applied Load Before and after Optimization

safe for operation. Stress distribution on the optimized plates is given in Fig. 11 for the three plates.

\section{Discussions}

Finite element-based topology optimization is a technique that is effectively used to design mechanical parts. In early studies, the effects of ERM on solution duration are investigated and these studies showed that ERM can decrease required solution duration for topology optimization up to $80 \%$ [10]. In this study, effectiveness of the method to obtain an optimum geometry for interbody implants is indicated. The topology optimization problem is formulated to select and eliminate minimum stressed elements, subject to a mass fraction constraint and a maximum allowable stress value. The optimum topology design comprises about $50 \%$ of the initial volume for the plate design. When the stress values of initial and optimized shape are compared in Table 1, maximum stress values are observed to have increased but the new stress values compared to yield value (200 MPa) are very low and displacement values are observed to be even lower after proper election of half of the material. Examining the stress distribution of optimized shape in Fig. 11, values are low even at the narrowest zones.

Common topology optimization algorithms do not control the effects of uncertainties such as materials, geometry, loading and etc. In the reliability-based topology optimization (RBTO) algorithms, these uncertainties can affect the solutions. Kharmanda et al. [16] investigated the effect of reliability-based analysis with topology optimization. In the present study, the maximum stress value after optimization appeared

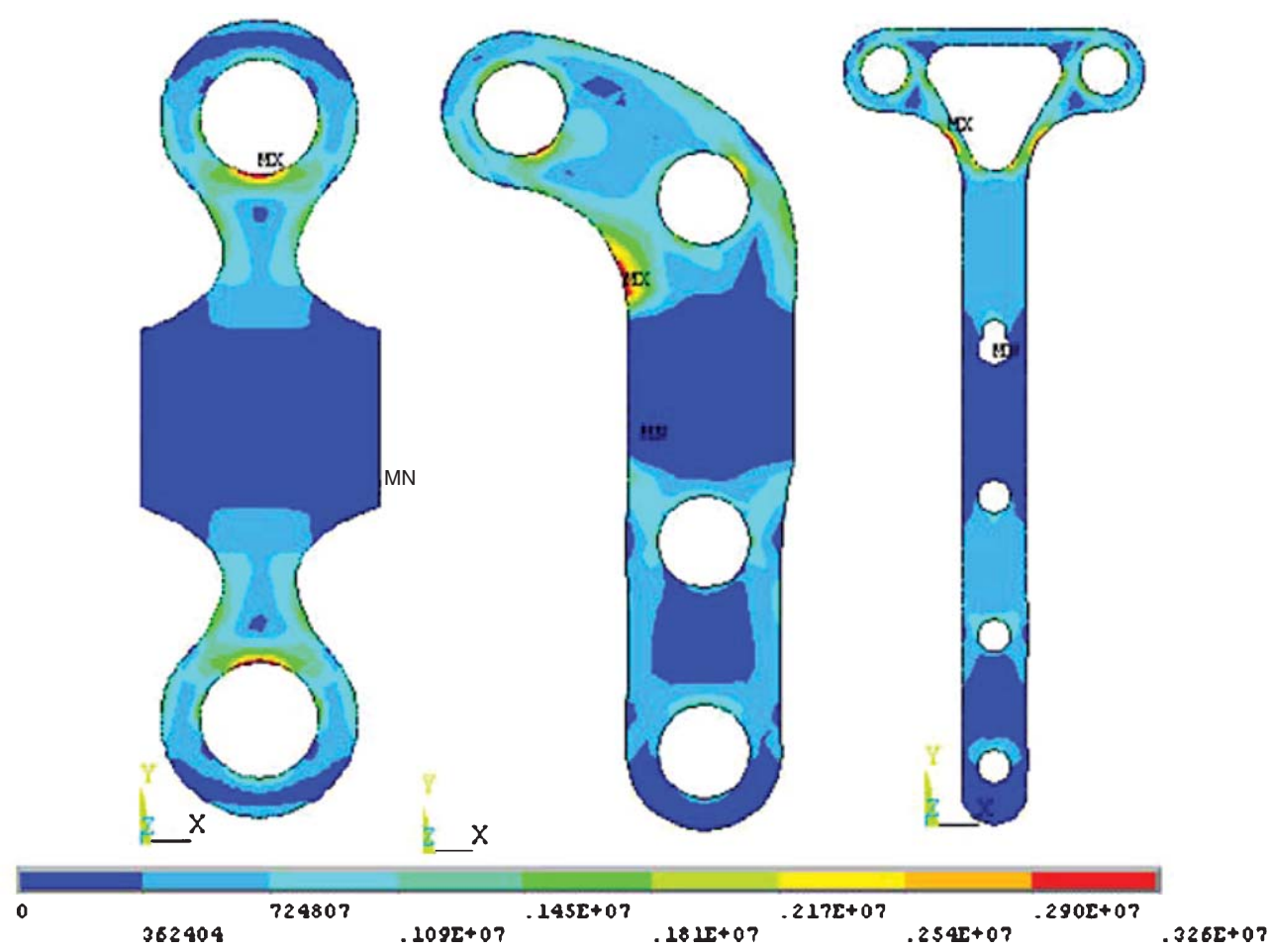

Fig. 11. Von Mises Stress Distribution on the Plates after Topology Optimization (Deformation Scale $=1 * 1000$ ). 
to be very low compared to the yield strength of the material, hence reliability-based analysis is not needed.

Application of topology optimization to interbody implants seems to be beneficiary. Especially if the implant is massive compared to the ones presented in the present work, topology optimization may prevent excessive material usage.

\section{References}

[1] M.H. Abolbashari, and S. Keshavarzmanesh, On various aspects of application of the evolutionary structural optimization method for 2D and 3D continuum structures, Finite Elements in Analysis and Design 42 (2006), 478-491.

[2] António A.-C., R. António and A. José, Simões, A model of bone adaptation as a topology optimization process with contact, J Biomedical Science and Engineering 5 (2012), 229-244.

[3] M.P. Bendsøe and N. Kikuchi, Generating optimal topologies in structural design using homogenization method, Computer Methods in Applied Mechanics and Engineering 71 (1988), 197-244.

[4] M.P. Bendsøe and O. Sigmund, Topology Optimization Theory, Methods and Applications, Berlin: Springer-Verlag, (2003).

[5] G. Chiandussi, I. Gaviglio and A. Ibba, Topology optimisation of an automotive component without final volume constraint specification, Advances in Engineering Software 35 (2004), 609-617.

[6] G.H. Chuah, A.R. Inzarulfaisham and M.I. Yusof, Topology optimization of spinal interbody cage for reducing stress shielding effect, Computer Methods in Biomechanics and Biomedical Engineering 13 (2010), 319-326.

[7] H.G. Chuah, I.A. Rahim and M.I. Yusof, Topology optimisation of spinal interbody cage for reducing stress shielding effect, Computer methods in biomechanics and biomedical engineering 3(13) (2010), 319-326.

[8] İ Esenkaya and N. Elmali, Proximal tibia medial open-wedge osteotomy using plates with wedges: Early results in 58 cases, Knee Surgery Sports Traumatology Arthroscopy 14 (2006), 955-961.

[9] İ Esenkaya, M. Misirlioglu, M.H. Kelestemur, N. Elmali and E. Fadillioglu, Biomechanical evaluation of different fixation plates in medial opening upper tibial osteotomy, The Knee 14 (2007), 46-50.

[10] İ Göv and M.A. Kütük, Topoloji optimizasyonunda eleman silme metodunun uygulanması15. Ulusal Mekanik Kongresi (2007), 437-446.

[11] A. Grujicic, M. Laberge, X. Xie, G. Arakere, B. Pandurangan, M. Grujicic, K.J. Jeray and S.L. Tanner, Computational investigation of the relative efficacies of nail- and plate-type proximal femoral-fracture fixation implants, Multidiscipline Modelling in Materials and Structures 4(6) (2010), 417-437.

[12] M. Grujicic, G. Arakere, X. Xie, M. LaBerge, A. Grujicic, D.W. Wagner and A. Vallejo, Design optimization and material selection for a femoral-fracture fixation-plate implant, Materials and Design 31 (2010) 3463-3473.

[13] M. Grujicic, X. Xie, G. Arakere, A. Grujicic, D.W. Wagner and A. Vallejo, Design-optimization and material selection for a proximal radius fracture-fixation implant, Journal of Materials Engineering and Performance 8(19) (2010), 1090-1103.

[14] J.K. Guest and J.H. Prevost, Optimizing multifunctional materials: Design of microstructures for maximized stiffness and fluid permeability, International Journal of Solids and Structures 43 (2006), 7028-7047.

[15] Z. Juan, L. Shuyao and L. Guangyao, The topology optimization design for continuum structures based on the element free Galerkin method, Engineering Analysis with Boundary Elements 34 (2010), 666-672.

[16] G. Kharmanda, N. Olhoff, A. Mohamedand and M. Lemaire, Reliability-based topology optimization, Struct Multidisc Optim 26 (2004), 295-307.

[17] M.S. Kuster, G.A. Wood, G.W. Stachowiak and A. Gachter, Joint load consideration in total knee replacement, Journal of Bone and Joint Surgery - British Volume 79 (1997), 109-113.

[18] U.D. Larsen, O. Sigmund and S. Bouwstra, Design and fabrication of compliant micromechanisms and structures with negative poisson's ratio, Journal of Microelectromechanical Systems 6(2), (1997), 99-106.

[19] C. Ledermann, P. Ermanni and R. Kelm, Dynamic CAD objects for structural optimization in preliminary aircraft design, Aerospace Science and Technology 10 (2006), 601-610.

[20] C.Y. Lin and S.H. Lin, Artificial neural network based hole image interpretation techniques for integrated topology and shape optimization, Comput Methods Appl Mech Engrg 194 (2005), 3817-3837.

[21] A. Mahdavi, R. Balaji, M. Frecker and E.M. Mockensturm, Parallel optimality criteria-based topology optimization design, 6th World Congresses of Structural and Multidisciplinary Optimization Rio de Janeiro (2005)

[22] H.P. Mlejnek, Some aspects of the genesis of structures, Structural Optimization 5 (1992), 64-69.

[23] Y.D. Seo, H.J. Kim and S.K. Youn, Shape optimization and its extension to topological design based on isogeometric analysis, International Journal of Solids and Structures 47 (2010), 1618-1640.

[24] D.G.T. Strange and S.T. Fisher, et al., Restoration of compressive loading properties of lumbar discs with a nucleus implant-a finite element analysis study, The Spine Journal 10 (2010), 602-609.

[25] A. Tovar, S.E. Gano, J.J. Mason and J.E. Renaud, Optimum design of an interbody implant for lumbar spine fixation, Advances in Engineering Software 36 (2005), 634-642.

[26] S.Y. Wang and K. Tai, Structural topology design optimization using Genetic Algorithms with a bit-array representation, Comput Methods Appl Mech Engrg 194 (2005), 3749-3770.

[27] Y.M. Xie and G.P. Steven, A simple evolutionary procedure for structural optimization, Computers \& Structures 49 (1993), 885-896.

[28] R.J. Yang and C.H. Chuang, Optimal topology design using linear programming, Computers \& Structures 52 (1994), 265-275. 

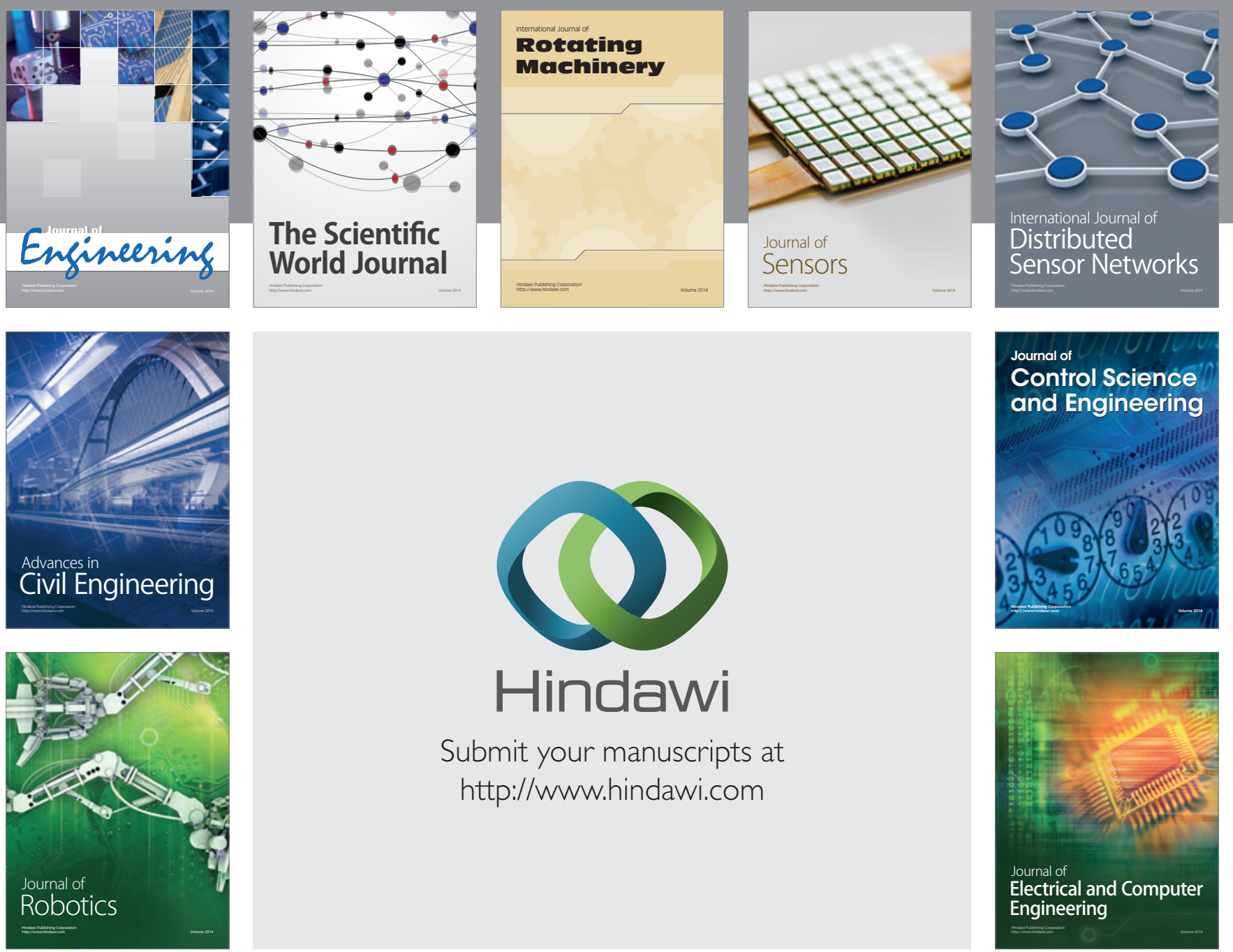

Submit your manuscripts at

http://www.hindawi.com
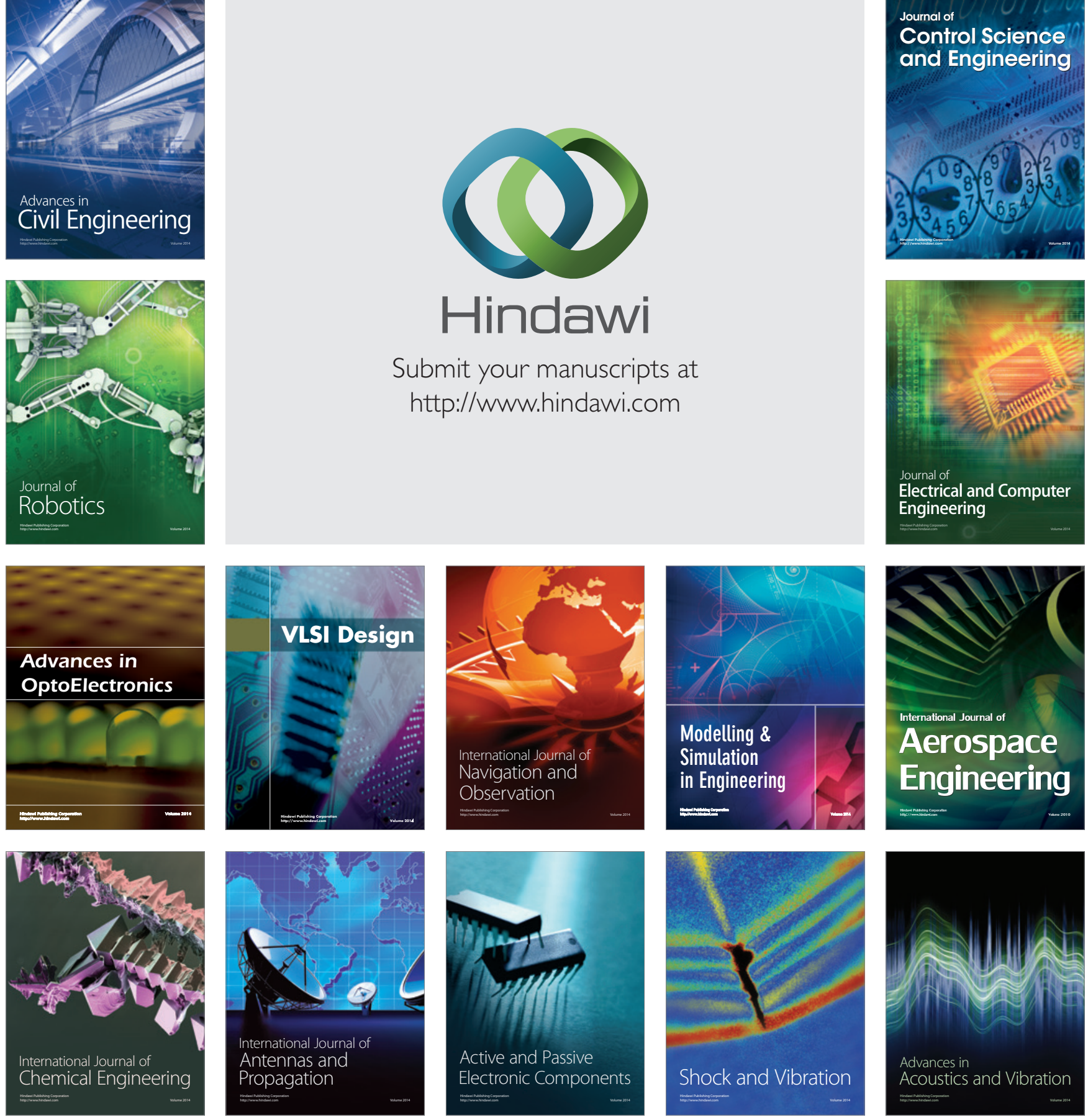